\title{
UN INFANTE DE “ELITE"WARI EN PUCLLANA: RESULTADOS DEL PROCESO DE DESENFARDADO
}

\author{
Gladys PAZ FLORES \\ Museo de Sitio Pucllana, Lima - Perú \\ pazflores.gladys@gmail.com
}

\section{RESUMEN}

Las últimas investigaciones realizadas en el Centro Ceremonial Pucllana, han dado a conocer la existencia de 82 tumbas pertenecientes a un cementerio de la época Wari ubicado entre la IV y VI Plataforma de la Gran Pirámide. En la temporada 2008, del Proyecto de Investigación, Conservación y Puesta en Valor Huaca Pucllana (PICPVHP) dirigido por la Dra. Isabel Flores Espinoza, se excavó un contexto funerario individual perteneciente a un infante que conservaba la mayor parte de sus elementos in situ como la estructura, el fardo y ofrendas. En la temporada 2011, se realizó el proceso de desenfardado a cargo de un equipo multidisciplinario entre arqueólogos, bioantropólogos y conservadores del cual se exponen en esta publicación; la metodología, características y composición del fardo y los resultados con el fin de entender a práctica funeraria Wari para los infantes de elite en la Costa Central.

\section{PALABRAS CLAVES}

Huaca Pucllana, Costa Central, Contextos funerarios, Wari.

\section{AN INFANT OF "ELITE"WARI IN PUCLLANA: RESULTS OF THE UNWRAPPING PROCESS}

\section{ABSTRACT}

The latest research carried out in the Pucllana Ceremonial Center, have revealed the existence of 82 tombs belonging to a cemetery of the Wari period located between the IV and VI Platform of the Great Pyramid. In the 2008 season, the Huaca Pucllana Research, Conservation and Valorization Project (PICPVHP) led by Dr. Isabel Flores Espinoza, excavated an individual funerary context belonging to an infant that retained most of its elements in situ as the structure, the bale and offerings. In the 2011 season, the unbundling process was carried out by a multidisciplinary team among archaeologists, bioanthropologists and curators of which are exposed in this publication; the methodology, characteristics and composition of the bale and the results in order to a better understanding of Wari funerary practice for infants of the elite social class in the Central Coast area.

\section{KEYWORDS}

Huaca Pucllana, Central Coast, Funerary contexts, Wari.

Recibido: $17 / 04 / 2020$

Aprobado: 18/06/2020 


\section{INTRODUCCIÓN}

$\mathrm{E}$

Centro Ceremonial Pucllana, se encuentra ubicado en el distrito de Miraflores, provincia de Lima, en la Costa Central del Perú. Actualmente tiene una extensión de 6 has y se compone arquitectónicamente de una pirámide constituida por siete plataformas y un Complejo Administrativo hacia el noreste compuesto por plazas y recintos construidos a base de adobitos de barro hechos a mano que datan de los años 450-700 d.C. pertenecientes a la Cultura Lima.

En 1981 se da inició al Proyecto de Investigación, Conservación y Puesta en Valor Huaca Pucllana bajo la dirección de la Dra. Isabel Flores Espinoza que viene trabajando hasta la actualidad a través de un convenio interinstitucional entre la Municipalidad de Miraflores y el Ministerio de Cultura.

En estos 39 años de investigación se ha recuperado valiosa información sobre las tres ocupaciones prehispánicas del sitio: La ocupación Lima (450 - 700 d.C.) que representa la arquitectura monumental expuesta, donde se producían las ceremonias y rituales; la ocupación funeraria Wari ubicado entre la IV y VI Plataforma de la Gran Pirámide (800-1000 d.C.); y las ofrendas tardías ubicadas en la parte baja de la ladera oeste y contextos funerarios en la cima de la Gran Pirámide pertenecientes a la sociedad Ychsma (1100-1452 d.C.).

Desde el año 2005, se han realizado importantes hallazgos correspondientes a contextos funerarios de la época Wari, pertenecientes a diversos personajes como pescadores, tejedoras, la tumba del sacerdote e infantes de elite, uno de estos hallazgos es el que se presenta en esta publicación.

Este hallazgo corresponde a un contexto funerario individual, de carácter intacto compuesto por un fardo de infante en buen estado de conservación y sus objetos asociados externos (ofrendas). El proceso de desenfardado fue realizado en los gabinetes del Museo de Sitio Pucllana a cargo del equipo multidisciplinario de arqueólogos, bioantropólogos y conservadores del proyecto con el objetivo de recuperar la mayor información del proceso en base a un registro riguroso de forma escrita, gráfica y fotográfica durante toda la intervención, que consistió en el retiro de las capas del fardo, de manera ordenada y forma inversa a como fueron depositados. El resultado de este trabajo se expone de la misma manera estructurada en que se realizó la investigación.

\section{Ocupación funeraria Wari de Pucllana}

La ocupación funeraria Wari de Pucllana se ubica temporalmente en la época 2B-3 del Horizonte Medio, entre los 800 dc. y 1000 dc. Hasta el momento se han identificado 82 contextos funerarios distribuidas entre la IV y VI Plataforma, en la cima de la Gran Pirámide. La mayor parte de estas tumbas han sufrido alteraciones parciales y totales debido a la profanación y re entierro de las mismas. 


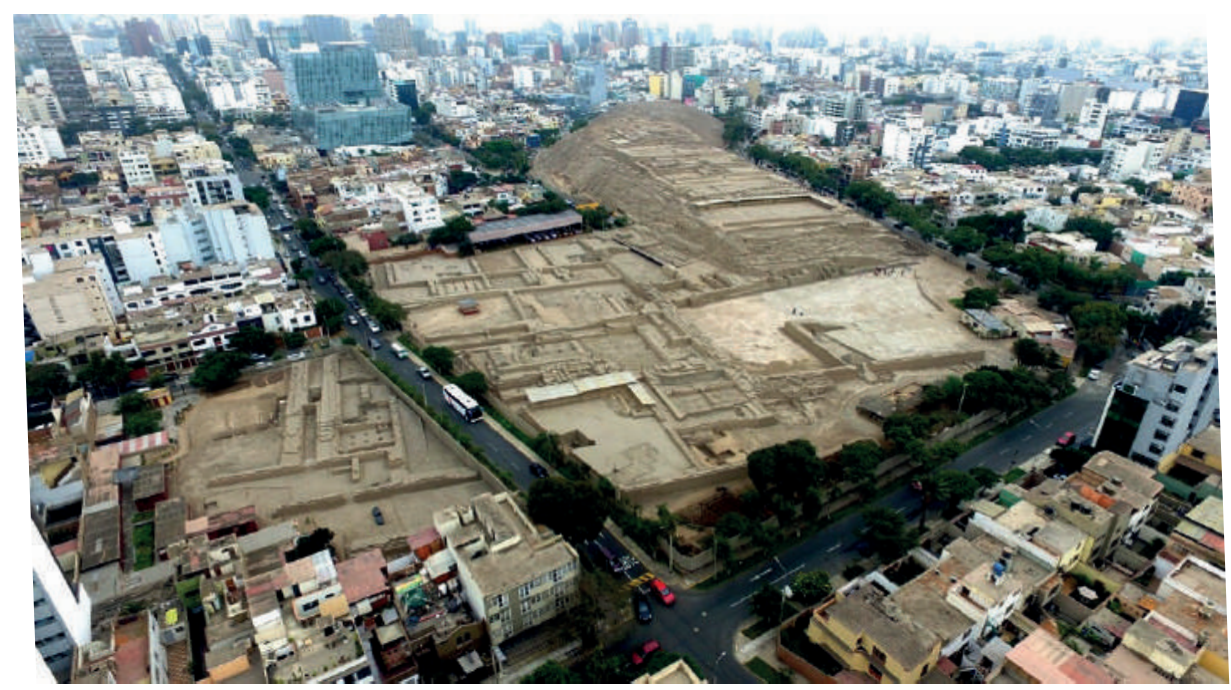

Figura 1. Centro Ceremonial Huaca Pucllana

Para la construcción de las tumbas, los Wari tuvieron que destruir y aprovechar parte de los elementos arquitectónicos que los Lima dejaron expuestos. Sin embargo, esta forma de aprovechar el espacio para colocar sus tumbas se observa también en otros sitios de la Costa Central con las mismas características ${ }^{1}$.

Cada contexto se compone de una estructura funeraria que de acuerdo a sus características morfológicas corresponden a 4 tipos: Pozo, Cista, Cista pozo y Cámara (Flores, 2012:21). Entre los elementos internos de cada estructura se observan los fados funerarios y objetos asociados externos también llamados ofrendas funerarias. Las tumbas pueden ser individuales o múltiples según el número de individuos al interior. Flores y Ccencho (2013) han reconocido la existencia de una jerarquización entre los fardos funerarios de las tumbas múltiples, de acuerdo a las características externas (presencia de falsa cabeza, vestimenta y ornamentos del fardo), la disposición del fardo principal al interior de la tumba, se encuentra casi siempre al SO y orientados al NE. Además, se ha identificado una recurrencia en la presencia de grandes bloques de barro al interior de cada estructura, lo que probablemente responda a una especie de marcadores de tumba.

\section{Características de contexto funerario}

El contexto funerario fue excavado en la temporada 2008 del PICPVHP a cargo de la arqueóloga Edines Pebe, al que lo denomino CF 16(08) 2 . Se trata de una tumba primaria individual, de carácter intacto, ubicada hacia el lado este del acceso

1 En la Costa Central la presencia Wari se caracterizó principalmente a partir de los contextos funerarios que se reportaron en sitios de Ancón (Segura, 1997; Kaulicke, 1997), Cajamarquilla (Mogrovejo y Segura, 2000), Nievería (Segura, 2001), Huaca Pucllana (Flores, 2005; Durand, 2010), Catalina Huanca (Cornejo, 2007; Álvarez, 2011), Pachacamac (Kaulicke, 2000); por el norte, Cayan y Lauri (Villacorta y Tosso, 2000), Huarmey (Sandoval, 2013); al sur, en el valle de Asia, Huaca Malena (Ángeles et al, 2004).

2 Véase Informe final de las actividades realizadas por el Proyecto de Investigación, Conservación y puesta en valor Huaca Pucllana 2008 presentado al Ministerio de Cultura, Lima. (Flores, 2009). 
correspondiente al pasadizo que divide la IV y V plataforma, parte superior central de la Gran Pirámide entre las coordenadas UTM N: 8660316.0406 E: 278643.2971.

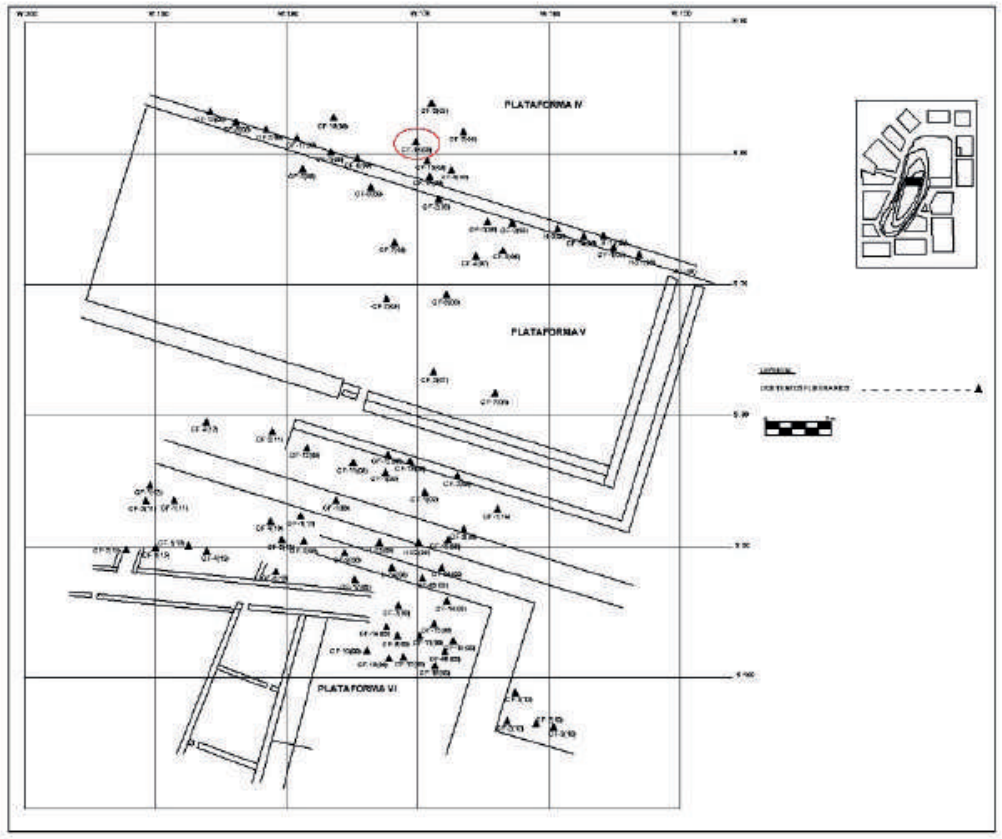

Figura 2. Ubicación del Contexto Funerario CF 16(08)

Para Kaulicke (1997:25), la identificación de un contexto funerario se basa en tres elementos: a) la estructura, b) el individuo y c) los objetos asociados. En este sentido, este hallazgo se compone de una estructura de tipo pozo ${ }^{3}$ de $55 \mathrm{~cm}$. de diámetro y $40 \mathrm{~cm}$ de profundidad a 107.77 m.s.n.m. Al interior se registraron dos subniveles estratigráficos:

El primer subnivel lo compone la matriz de la fosa funeraria hallada en medio del relleno de clausura del pasadizo, un depósito compuesto de cantos rodados y tierra suelta que cubrían los elementos internos del contexto: el fardo y objetos asociados.

El fardo se encontró en posición vertical, sostenido y reforzado por medio de 9 adobitos dispuestos en sus cuatro lados que además cubrían las ofrendas externas: una vasija de mate y una bolsa elaborada en fibra de algodón, asociadas al rango social del individuo. (Paz, 2016)

El segundo subnivel, está representado por la base de la estructura, compuesta por una capa de barro de aproximadamente $5 \mathrm{~cm}$ de grosor que se dispone sobre un poste de madera, tronco de Prosopis pallida (Huarango), perteneciente a una fase constructiva anterior, el cual fue acondicionado, cortado y nivelado para la colocación del fardo y sus ofrendas.

3 Concepto tomado de la caracterización de estructuras funerarias (Flores, 2013: 35). 


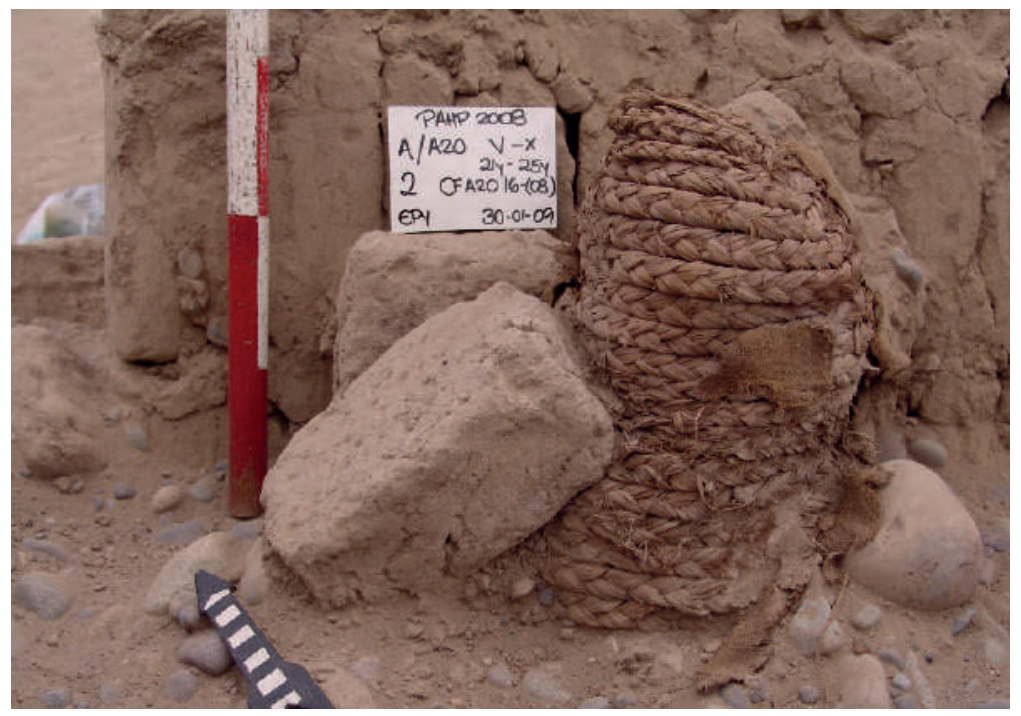

Figura 3. Contexto Funerario CF 16(08)

\section{METODOLOGÍA DE TRABAJO DEL PROCESO DE DESENFARDADO}

La metodología empleada para el desarrollo del proceso de desenfardado se encuentra bajo las líneas de investigación del PICPVHP, que tiene como principio básico realizar un registro detallado para reconstruir el ritual funerario a partir de la organización de los objetos.

Antes de la intervención, se sugiere la toma de placas radiográficas que nos indiquen el estado interno del fardo, así como la presencia de otros elementos a parte del individuo, con el fin de conocer la ubicación y considerar algunos aspectos antes de iniciar el proceso.

La intervención se realiza teniendo como base el principio estratigráfico, retirando y registrando cada capa desde la externa hacia la interna con el fin de mantener el orden inverso del procedimiento original y nos permita llegar a la reconstrucción total del mismo. Cada capa puede estar compuesta por uno o más elementos (textiles, vegetales, ornamentos, etc.), cada uno de estos, es registrado con las siglas EE (elemento especial) llevando un orden correlativo en la numeración, de acuerdo al inventario del proyecto.

El registro escrito, está compuesto por fichas y un cuaderno de campo que llevan las descripciones y dibujos de croquis y detalles que refuercen la información. Para el registro gráfico se realizan dibujos de planta, perfil y detalles en escalas 1/1 o 1/2 dependiendo del tamaño real de cada elemento, utilizando colores para la diferenciación de las leyendas. Además, se coloca un membrete en la parte inferior derecha con la información de cada gráfico. De igual manera, se lleva un orden en el registro fotográfico y de video, teniendo las tomas tanto generales como de detalles de cada capa descrita. 
El embalaje de los materiales recuperados en el proceso de desenfardado dependerá del estado de conservación de cada elemento, bajo la supervisión y manejo del conservador y/o restaurador encargado. Para ello, se utilizarán diferentes soportes y materiales que ayuden a la prevención del deterioro una vez expuesto, posteriormente son trasladados a los gabinetes correspondientes para el siguiente proceso de trabajo a cargo de cada especialista.

\section{El Fardo Funerario}

El fardo presenta una forma tronco-cónica de $33 \mathrm{~cm}$ de alto por $25 \mathrm{~cm}$ de diámetro dispuesto de forma vertical. Antes de la intervención, se tomaron 2 placas de Rayos X (frontal y lateral derecho), con el objetivo de conocer la disposición del individuo y de los elementos al interior.

En el informe presentado por el Dr. Alfonso Onofre del EMETAC (Centro Especializado en Diagnóstico por Imágenes), concluye que se trata de un fardo funerario pediátrico con una edad cronológica menor a 3 meses, donde la mayoría de los restos óseos se encuentran desarticulados, sin embargo, por la posición del fémur es probable que el infante se encontrara en posición flexionado. Entre las características externas sobresale el uso de soguillas de fibra de totora elaboradas en técnica trenzada, que lo envuelven totalmente de forma espiral desde la parte inferior a la superior, sujetas por medio de un hilo de cabuya y cubiertas por un fragmento textil en la parte superior que podría ser considerada como parte de la vestimenta del fardo.

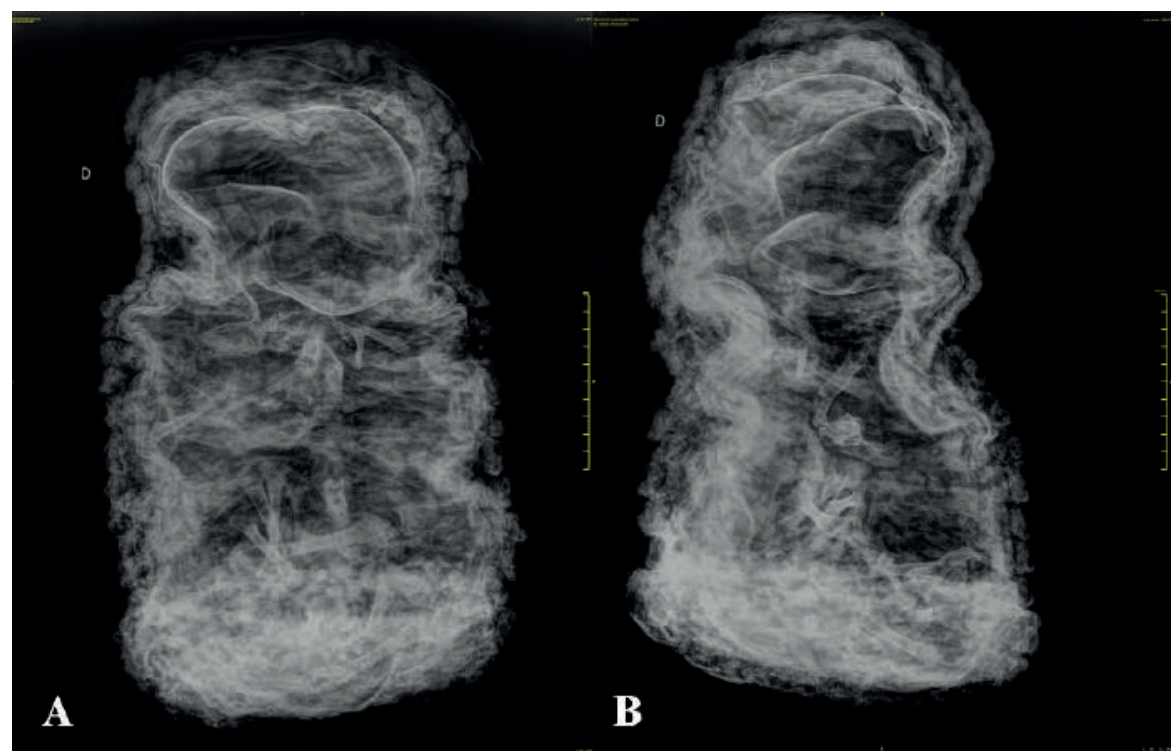

Figura 4. Radiografía del Fardo Funerario vista frontal 


\section{Composición del fardo}

Como resultado del proceso de desenfardado se registraron 8 capas compuestas de diferentes elementos; entre fragmentos textiles, soguillas de material orgánico y cuero de camélido:

Capa 1.- Envoltorio externo compuesto por dos elementos:

El elemento (EE 01), fragmento de tejido dispuesto en la parte superior del fardo, sobre las soguillas externas (EE 02), probablemente forme parte de la vestimenta del fardo.

Las soguillas (EE 02) se dispone a lo largo del fardo desde la parte inferior hacia la parte superior, con un total de 20 vueltas en forma de espiral y en sentido anti horario, sujetadas por medio de un hilo de cabuya.

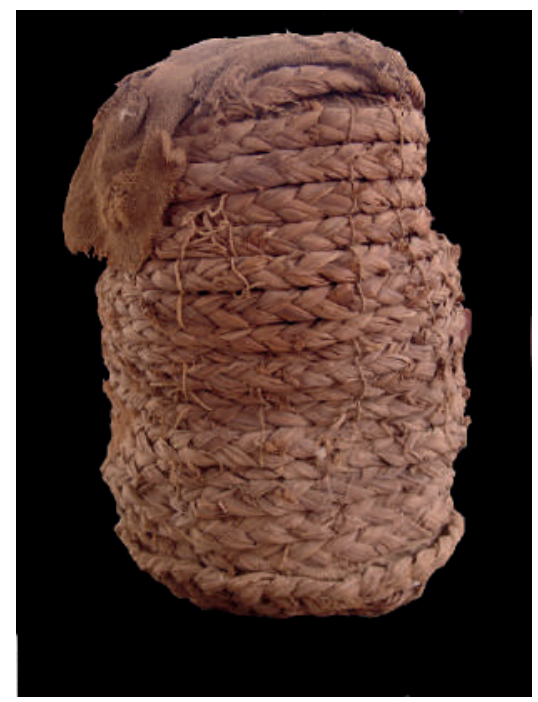

Figura 5. Fardo de infante. Capa 1: Quinto envoltorio

Capa 2.- Conforma parte del relleno del fardo, se compone de un fragmento de tejido (EE 03) colocado hacia el lado izquierdo del fardo, cubriendo la parte inferior de las soguillas pertenecientes a la siguiente capa.

Capa 3.- Consiste en dos fragmentos de tejido (EE 04), que fueron previamente doblados y colocados como base alrededor del fardo con el fin de darle estabilidad y mantener su posición vertical. Posterior al retiro de este elemento, el fardo funerario se coloca de forma horizontal para continuar con la intervención.

Capa 4.- Se trata del cuarto envoltorio del fardo, conformado por una red de soguillas (EE 05) que da consistencia al paquete y asegura las capas internas del fardo funerario. 


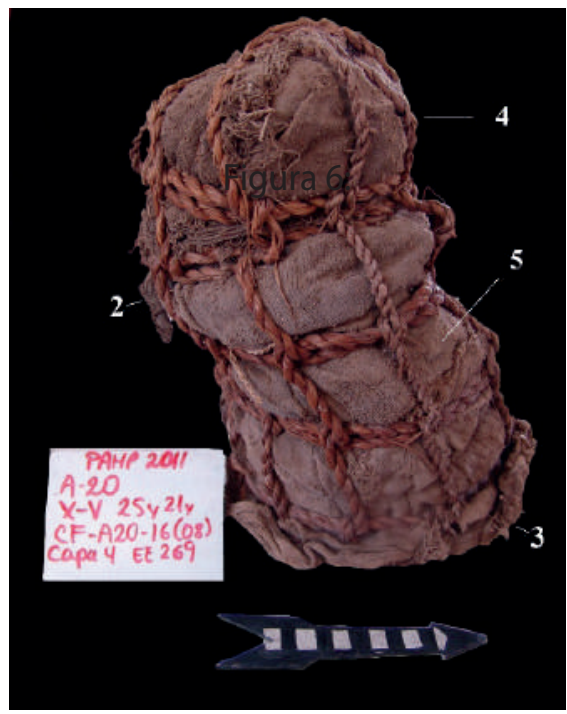

Figura 6. Capas 2: Relleno, Capa 3: Base, Capa 4: Cuarto Envoltorio y Capa 5 Tercer envoltorio.

Capa 5.- Es el tercer envoltorio, consiste en un tejido (EE 06) compuesto por 3 paños cosidos que le dan una forma rectangular, se coloca de forma vertical que cubre al infante completamente de atrás hacia adelante, posteriormente es atado por soguillas delgadas elaboradas en fibra de cabuya y en técnica de torsión, en la parte superior y en la parte central.

Capa 6.- Se trata del segundo envoltorio del fardo, conformado por una faja (EE 09) que envuelve el fardo por medio de 6 vueltas en sentido anti horario, desde la parte superior hacia la parte inferior. La última vuelta va de regreso formando una especie de ojal donde se introduce uno de los extremos y se anuda por medio de 2 lazadas. En la parte central se halló otro fragmento de tejido (EE 10).

Capa 7.- Primer envoltorio del fardo, se compone de dos elementos: Un tejido (EE 11) que cubre al infante totalmente y es atado por medio de una vincha (EE 12) a la altura del cráneo. El envoltorio ha sido colocado desde la parte posterior hacia la anterior, posteriormente es atado por medio del (EE12) a la altura de la cabeza donde el amarre del nudo simple está en la cara anterior.

Capa 8: Se compone de los elementos que conforman el tratamiento del cadáver antes de iniciarse el proceso de desenfardado: El infante llevaba puesta una prenda que, según sus características morfológicas, indican que se trata de un unku, prenda utilizada generalmente por el género masculino (Chuchon 2013: 146). Sobre la parte frontal del cráneo se disponía un fragmento de cuero animal, probable camélido, cubriendo completamente el cráneo. Además, se hallaron soguillas en fibra de cabuya que entrelazaban las falanges superiores e inferiores. 


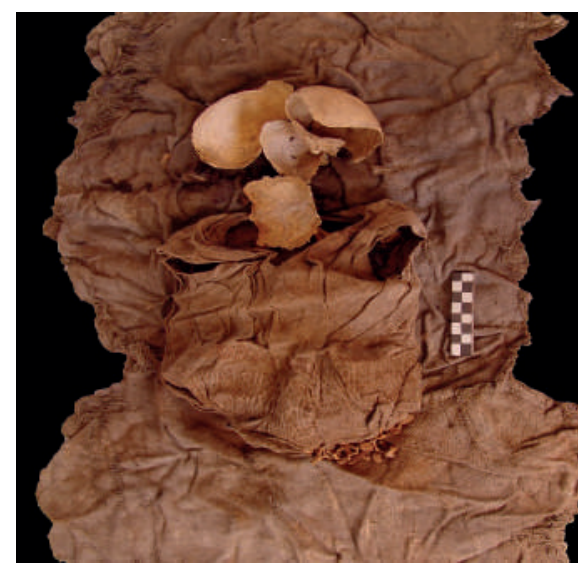

Figura 7. Capa 7: Primer envoltorio y tratamiento del cadáver.

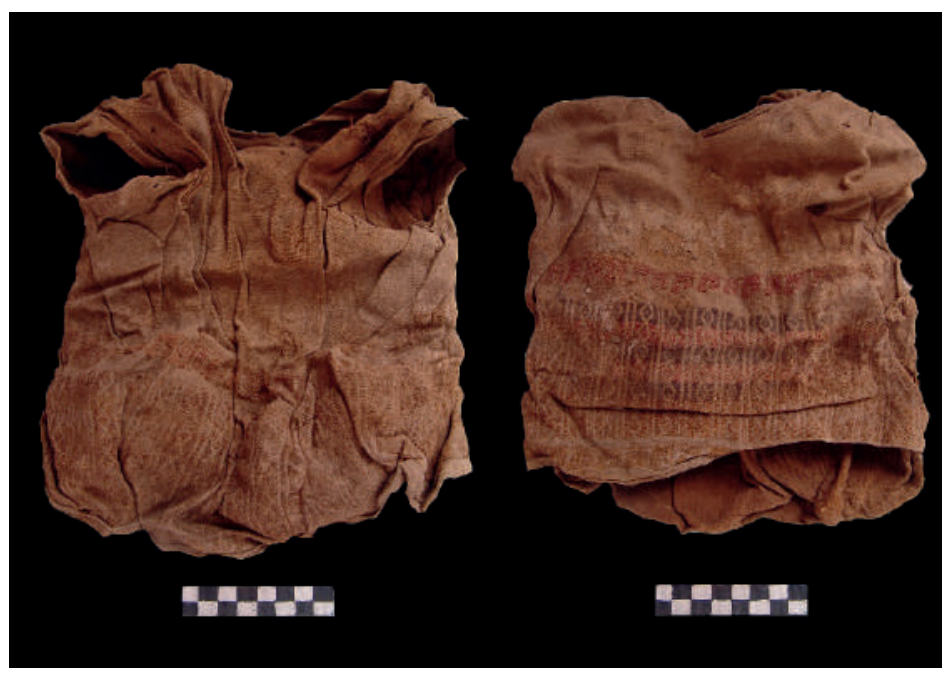

Figura 8. Vestimenta del infante

La posición del individuo al interior del fardo corresponde a la posición fetal, con ambas extremidades flexionadas y las manos a la altura del rostro.

\section{Conservación preventiva de los materiales:}

Los trabajos de conservación y restauración del material textil y orgánico están a cargo de los especialistas del proyecto y se realizan posteriormente al proceso de desenfardado en los gabinetes respectivos. Sin embargo, en esta intervención se consideró trabajar paralelamente en la conservación preventiva de las soguillas externas (Capa 1) e internas (Capa 4) con el fin de reproducir el proceso de enfardamiento del infante a detalle y mantener su posición original. 
Antes de retirar ambas soguillas se construyeron dos soportes acondicionados, uno de tela de yute y otro de tela de tipo guata de las mismas dimensiones del fardo, subsiguientemente las soguillas se fueron colocando de manera inversa con el objetivo de rescatar los detalles en la manera en que fueron colocadas. Finalmente, estos fueron entregados al especialista de conservación y restauración de material orgánico para su respectivo tratamiento.

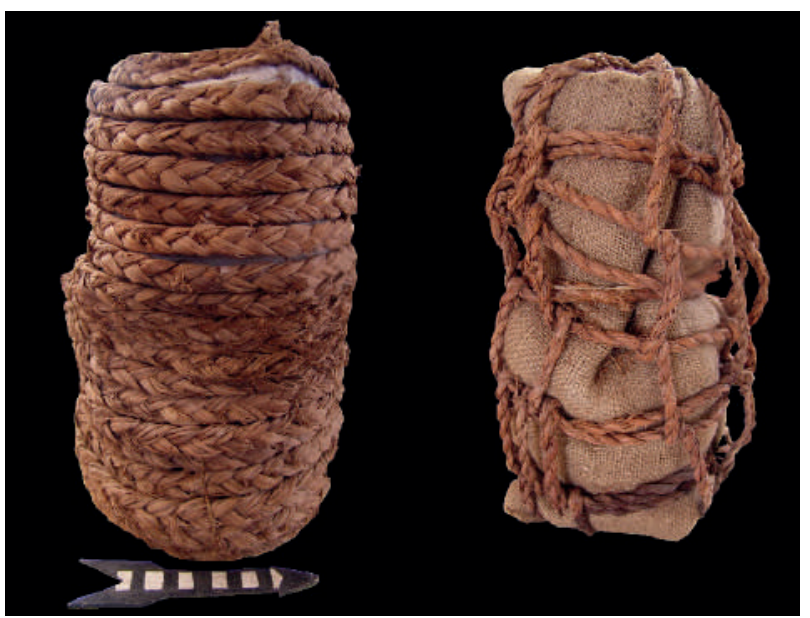

Figura 9. Conservación preventiva

\section{RESULTADOS}

El objetivo principal de este trabajo es reconocer la práctica funeraria Wari en Pucllana, y los actos rituales a través del tratamiento de la muerte. Para ello, se debe de considerar los procesos tafonómicos del contexto funerario previos al proceso de desenfardado, con el fin de que nos indicara el estado del fardo para entender e interpretar correctamente la naturaleza del contexto (Ortiz, 2011). Por ello, consideramos exponer en la primera parte, los datos recogidos de la exhumación del infante:

1) Características externas del contexto funerario

La distribución de los contextos funerarios Wari nos muestra una concentración de tumbas asociadas a los muros perimetrales de tipo cámara y otro grupo que intruye elementos arquitectónicos como pisos, banquetas y rellenos donde se consideran los tipos cista o pozo. Este contexto en particular presenta estructura de tipo pozo, intruía al relleno de clausura compuesto de tierra y cantos rodados del pasadizo que divide la IV y $\mathrm{V}$ plataforma. La distribución al interior de la fosa nos indica que el espacio reducido fue previamente planificado y elaborado especialmente para el infante ${ }^{4}$ y sus ofrendas.

4 Se ha considerado la denominación infante, de nacimiento a 3 años de edad, tomado de Buikstra y Ubelaker(1994). 
2) Características internas: Tratamiento del cadáver

Se registraron 8 capas en el proceso de desenfardado, desde los elementos que conforman la preparación del individuo hasta el envoltorio externo:

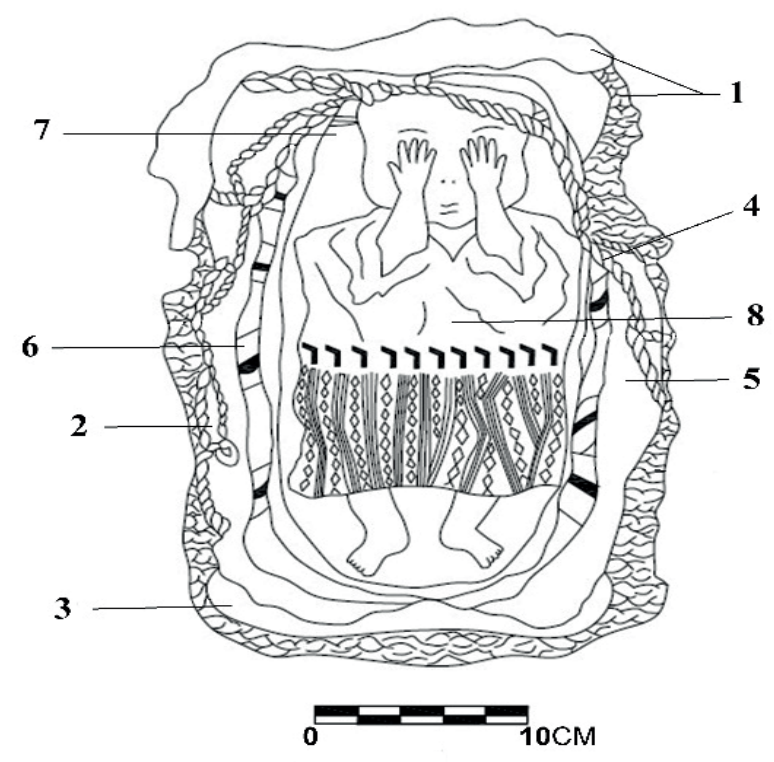

Figura 10. Dibujo reconstructivo del fardo

Tabla 1. Composición del fardo funerario

\begin{tabular}{|c|c|c|c|c|}
\hline Capa & Denominación & Elemento & Descripción & Características \\
\hline \multirow[t]{2}{*}{1} & \multirow[t]{2}{*}{$\begin{array}{l}\text { Quinto } \\
\text { envoltorio }\end{array}$} & EE 01 & $\begin{array}{l}\text { Fragmento } \\
\text { de paño }\end{array}$ & $\begin{array}{l}\text { Disposición: Cubre la parte superior } \\
\text { del infante } \\
\text { Fibra: pelo de camélido } \\
\text { Técnica: cara de urdimbre } \\
\text { Color: marrón } \\
\text { Decoración: listada } \\
\text { Medidas: } 33 \mathrm{~cm} \times 17 \mathrm{~cm} . \\
\end{array}$ \\
\hline & & EE 02 & $\begin{array}{l}\text { Soguillas } \\
\text { trenzadas }\end{array}$ & $\begin{array}{l}\text { Disposición: Cubre totalmente al } \\
\text { infante } \\
\text { Fibra: totora y cabuya } \\
\text { Técnica: trenzada } \\
\text { Medida: } 12.58 \mathrm{~m} \text {. }\end{array}$ \\
\hline 2 & Relleno & EE 03 & $\begin{array}{l}\text { Fragmento } \\
\text { de paño }\end{array}$ & $\begin{array}{l}\text { Disposición: Cubre la parte inferior del } \\
\text { infante } \\
\text { Fibra: pelo de camélido } \\
\text { Técnica: cara de urdimbre } \\
\text { Color: marrón } \\
\text { Decoración: listada } \\
\text { Medidas: } 35 \mathrm{~cm} \times 25 \mathrm{~cm} \text {. }\end{array}$ \\
\hline
\end{tabular}




\begin{tabular}{|c|c|c|c|c|}
\hline 3 & Base & EE 04 & $\begin{array}{l}\text { Fragmento } \\
\text { de paño }\end{array}$ & $\begin{array}{l}\text { Disposición: base del fardo } \\
\text { Fibra: algodón } \\
\text { Técnica: cara de urdimbre } \\
\text { Color: pardo } \\
\text { Medidas: } 25 \mathrm{~cm} \times 25 \mathrm{~cm} \text {. } \\
\end{array}$ \\
\hline 4 & $\begin{array}{l}\text { Cuarto } \\
\text { envoltorio }\end{array}$ & EE 05 & $\begin{array}{l}\text { Soguillas } \\
\text { en torsión }\end{array}$ & $\begin{array}{l}\text { Disposición: Cubre totalmente al } \\
\text { infante } \\
\text { Fibra: totora } \\
\text { Técnica: torsión }\end{array}$ \\
\hline \multirow{3}{*}{5} & \multirow{3}{*}{$\begin{array}{l}\text { Tercer } \\
\text { envoltorio }\end{array}$} & EE 06 & $\begin{array}{l}\text { Fragmento } \\
\text { de paño }\end{array}$ & \multirow{3}{*}{$\begin{array}{l}\text { Disposición: Cubre la parte inferior del } \\
\text { infante } \\
\text { Fibra: algodón } \\
\text { Técnica: cara de urdimbre } \\
\text { Color: pardo } \\
\text { Medidas: } 90 \mathrm{~cm} \text { x } 46 \mathrm{~cm} .\end{array}$} \\
\hline & & EE 07 & $\begin{array}{l}\text { Soguillas } \\
\text { (totora) en } \\
\text { torsión } \\
\end{array}$ & \\
\hline & & EE 08 & \begin{tabular}{|l|} 
Soguillas \\
(totora) en \\
torsión
\end{tabular} & \\
\hline \multirow[t]{2}{*}{6} & \multirow{2}{*}{$\begin{array}{l}\text { Segundo } \\
\text { envoltorio }\end{array}$} & EE 09 & Faja & $\begin{array}{l}\text { Disposición: Cubre completamente al } \\
\text { infante. } \\
\text { Fibra: algodón } \\
\text { Técnica: cara de urdimbre } \\
\text { Color: pardo } \\
\text { Decoración: listada de color negro } \\
\text { Medidas: } 3.10 \mathrm{~m} \times 7 \mathrm{~cm} \text {. }\end{array}$ \\
\hline & & EE 10 & $\begin{array}{l}\text { Fragmento } \\
\text { de paño }\end{array}$ & $\begin{array}{l}\text { Disposición: Cubre la parte central del } \\
\text { infante } \\
\text { Fibra: algodón } \\
\text { Técnica: cara de urdimbre } \\
\text { Color: pardo } \\
\text { Medidas: } 40 \mathrm{~cm} . \text { x } 40 \mathrm{~cm} .\end{array}$ \\
\hline \multirow{2}{*}{7} & \multirow{2}{*}{$\begin{array}{l}\text { Primer } \\
\text { envoltorio }\end{array}$} & EE 11 & Vincha & $\begin{array}{l}\text { Disposición: Cubre la parte superior } \\
\text { del infante } \\
\text { Fibra: algodón } \\
\text { Técnica: cara de urdimbre } \\
\text { Color: pardo } \\
\text { Medidas: } 93 \mathrm{~cm} . \times 4 \mathrm{~cm} .\end{array}$ \\
\hline & & EE 12 & $\begin{array}{l}\text { Fragmento } \\
\text { de paño }\end{array}$ & $\begin{array}{l}\text { Disposición: Cubre la parte superior } \\
\text { del infante } \\
\text { Fibra: algodón } \\
\text { Técnica: cara de urdimbre } \\
\text { Color: pardo } \\
\text { Medidas: } 93 \mathrm{~cm} . \times 4 \mathrm{~cm} \text {. }\end{array}$ \\
\hline \multirow{4}{*}{8} & \multirow{4}{*}{$\begin{array}{l}\text { Preparación } \\
\text { del } \\
\text { individuo }\end{array}$} & EE 13 & $\begin{array}{l}\text { Cuero de } \\
\text { camélido }\end{array}$ & $\begin{array}{l}\text { Disposición: Cubre la parte superior } \\
\text { del infante } \\
\text { Fibra: cuero de camélido } \\
\text { Color: melange (marrón - blanco) } \\
\text { Medidas: } 18 \mathrm{~cm} . \times 13 \mathrm{~cm} \text {. } \\
\end{array}$ \\
\hline & & EE 14 & $\begin{array}{l}\text { Vestimenta } \\
\text { - Unku }\end{array}$ & $\begin{array}{l}\text { Fibra: algodón } \\
\text { Color: pardo } \\
\text { Técnica: cara de urdimbre } \\
\text { Decoración: Figuras geométricas en } \\
\text { técnica de brocado y color marrón y } \\
\text { rojo } \\
\text { Medidas: } 30 \mathrm{~cm} \text { x } 25 \mathrm{~cm} \text {. }\end{array}$ \\
\hline & & \begin{tabular}{|l|} 
EE 15 \\
\end{tabular} & \begin{tabular}{|l|} 
Soguillas \\
\end{tabular} & \multirow{2}{*}{ Fibra: Cabuya } \\
\hline & & EE 16 & Soguillas & \\
\hline
\end{tabular}

Fuente: Elaboración propia. 
La mayoría de los elementos textiles que componen el fardo son fragmentos de tejidos reutilizados, que han sido colocados como relleno y envoltorios internos, algunos atados por medio de soguillas que están elaboradas en totora y cabuya como materia prima y en las técnicas: trenzado y torsión. También se observan soguillas entrelazando las falanges de las manos y pies. Además, se hallaron una faja y una vincha, ambas dispuestas también como amarre de diferentes envoltorios colocadas en sentido anti horario. Existe una recurrencia en cuanto a la forma de asegurar los envoltorios mediante amarres que van en sentido anti horario también para otros contextos Wari de Pucllana.

El fardo funerario no presenta capa de vegetales ni falsa cabeza a diferencia de otros fardos considerados infante de jerarquía (Flores 2013: 44). Sin embargo, el infante lleva puesto un unku decorado, usado por el género masculino de élite (Chuchon, 2013:149) y que lo diferencia de los niños considerados sacrificados, los cuales no llevan vestimenta (Ccencho, 2013:127). Otra diferencia con los niños sacrificados es la presencia de una capa compuesta de cuero de camélido que cubre el rostro del infante similar a las que llevan los fardos principales.

3) Análisis bioantropológico:

El análisis bioantropológico realizado por la Mg. María Inés Barreto concluyó que se trataba de un esqueleto casi completo y bien preservado de un infante de 3 a 9 meses de edad al morir, de sexo indeterminado. El cuerpo no presenta lesiones osteopatológicas ni relacionadas al momento de la muerte. (Flores, 2012)

\section{DISCUSIÓN}

Para discutir el tema se han tomado en cuenta las recurrencias y diferencias entre los contextos funerarios de infantes Wari de Huaca Pucllana y otros sitios de la época en la Costa Central, con el fin de obtener los indicadores que caractericen el ritual funerario y status social del infante.

Flores y otros $(2012)$ y Flores $(2013,2016)$ han caracterizado los contextos funerarios Wari de Huaca Pucllana y define esta ocupación como un cementerio de élite. En relación a las características de la categoría infante de élite, la edad del individuo es menor a la señalada por Flores (2013), además la preparación del fardo difiere a la de un adulto, por la ausencia del relleno de capa de vegetales y falsa cabeza. Sin embargo, este infante presenta otras características que tampoco lo coloca dentro de la categoría de niño sacrificado.

- La tumba fue planificada y construida como individual, en ningún momento se tuvo la intencionalidad de pertenecer a un grupo al que se le pueda atribuir la categoría de acompañante o de un sacrificio hacia un individuo principal. De igual manera es difícil atribuirle una categoría por no formar parte de una tumba múltiple. 
- El fardo se encontraba orientado hacia el norte, dispuesto intencionalmente de manera vertical con sus propias ofrendas ubicadas en el lado este, mientras que los niños sacrificados son dispuestos de manera horizontal al interior de una tumba múltiple sin ninguna orientación en especial.

- La presentación externa del fardo se compone de un tejido, probable vestimenta del fardo, y el uso de soguillas gruesas trenzadas que van envolviendo al fardo de forma espiral desde la base hacia la parte superior, técnica similar a los fardos principales de adultos.

En el caso de otros infantes, se ha observado el uso de soguillas delgadas en la técnica de trenzado o torsión dispuesta de manera reticular sobre un envoltorio textil, siendo este el único con esta característica.

- El fardo del infante se conforma de paños de tejido reutilizados colocados como rellenos y envoltorios, sin embargo, vestía un unku decorado donde se diferencia notablemente el tipo de manufactura de calidad.

- Los resultados del análisis bioantropológico señala la ausencia de lesiones perimortem a diferencia de los niños sacrificados, donde los niños eran matados con golpes, principalmente en la cabeza (Ccencho 2013: 141).

- La preparación del infante previo al entierro, nos indica una planificación y conocimiento sobre el proceso de enfardamiento por parte de un especialista o sepultero ${ }^{5}$ además, del status social del infante dentro de la población.

\section{CONCLUSIONES}

La ocupación funeraria Wari de Pucllana es uno de los pocos sitios excavados casi en su totalidad en el valle bajo del Rímac, lo cual nos ofrece mayores indicadores para entender la práctica funeraria y reconocer la inversión de trabajo que se observa en las características de los elementos que componen los contextos funerarios y la importancia que esta tenia para la sociedad de la época.

En relación a otras ocupaciones funerarias Wari, se observa que en Lauri, las tumbas más elaboradas corresponden a niños (Villacorta y Tosso 2000: 112). En Catalina Huanca (Cornejo, 2009; Álvarez: 2011:32) señalan que los infantes si bien no presentan ofrendas internas (OAI), la presencia de ofrenda externas (OAE) relacionados a mates, ofrendas vegetales y minerales, se debe a una función discriminante con respecto al resto del grupo de edad. En Ancón y Huallamarca se observa una distinción en la posición de los infantes de acuerdo a la edad, los menores de 1.5 años se encuentran extendidos mientras que los mayores, en posición flexionada. (Tomado de Flores, 2013: 45). Estas recurrencias y diferencias encontradas, nos demuestra que el orden de los elementos que comprenden los contextos funerarios de infantes, ha sido demarcado por un ritual funerario diferenciado. En este caso en particular, existe una intención de mantener al

5 Concepto tomado de Flores (2013:81) 
infante en una posición flexionada y vertical, al igual que la presentación externa del fardo, similar a un fardo principal adulto de elite.

Finalmente, hemos seguido la línea de categorización inicial para llegar a una interpretación que nos acerque a entender a práctica funeraria Wari para los infantes de elite. Sin embargo, creemos que se debería de mostrar un panorama general de la complejidad de la ocupación funeraria Wari en la Costa Central con el fin de reevaluar los indicadores que nos lleven a reinterpretar nuevas categorías basadas en el estatus social tanto en infantes como en adultos.

Agradecimientos: En primer lugar, quiero agradecer a la Dra. Isabel Flores Espinoza, directora del Museo de Sitio Pucllana por su constante motivación a la producción de la investigación y por autorizarme el uso de los datos para esta publicación. También a todo el equipo de trabajo del PICPVHP desde la temporada 2009 hasta la actualidad por sus aportes y críticas a lo largo de la investigación, especialmente al Arqlo. José Ccencho por ser parte importante de mi formación. Finalmente, a mis amigos y colegas por el tiempo que se tomaron de revisar este artículo antes de su presentación.

\section{REFERENCIAS BIBLIOGRÁFICAS}

Álvarez, M. (2011). Contextos funerarios del Horizonte Medio en el Montículo 6 de Catalina Huanca. Una aproximación biológica y arqueológica. Tesis para optar el título de Máster en Antropología Física: Biodiversidad y Evolución Humana en la Universidad Autónoma de Madrid. Universidad Complutense. Universidad de Alcalá de Henares. España.

Buikstra JE, and Ubelaker DH. (1994). Standards for Data Collection from Human Skeletal Remains: Proceedings of a Seminar at the Field Museum of Natural History (Arkansas Archeological Report Research Series). Arkansas Archeological Survey.

Chuchon. H. (2013). Análisis de los tejidos encontrados en la tumba del sacerdote en Los Wari en Pucllana, la tumba de un sacerdote. Parte III, pp. 142-230. Ministerio de Cultura, Lima.

Ccencho, J. (2013). Excavación de la tumba del Sacerdote en Los Wari en Pucllana, la tumba de un sacerdote. Parte II, pp. 89-141. Ministerio de Cultura, Lima.

Cornejo, M. (2007). Informe Final del Proyecto de Evaluación Arqueológica Catalina Huanca. Lima. Presentado al Instituto Nacional de Cultura.

(2009). Informe Final del Proyecto de Rescate Arqueológico Catalina Huanca. Lima. Presentado al Instituto Nacional de Cultura.

Flores, I. (2005). Pucllana: Esplendor de la Cultura Lima. Instituto Nacional de Cultura. Lima. 
(2009). Informe final de las actividades realizadas por el Proyecto de Investigación, Conservación y Puesta en Valor Huaca Pucllana 2008. Presentado al Instituto Nacional de Cultura. Lima.

(2012). Informe final de las actividades realizadas por el Proyecto de Investigación, Conservación y Puesta en Valor Huaca Pucllana 2011. Presentado al Instituto Nacional de Cultura. Lima.

Flores, I. (compil.) (2013). Los Wari en Pucllana, la tumba de un sacerdote. Ministerio de Cultura, Lima.

Flores, Isabel (2016). "Avance de las Excavaciones Arqueológicas en la Gran Pirámide de Huaca Pucllana - Temporada 2014" En: Actas del I Congreso Nacional de Arqueología. Pp 141. Ministerio de Cultura.

Flores, l.; Chuchon, H.; Ccencho, J.; Vargas, P. (2012). La Doble Tela de los Muertos, Tejidos Especiales Wari de Pucllana. Auqui ediciones. Proyecto de Investigación, Conservación y Puesta en Valor Huaca Pucllana. Lima.

García L. (2013). Milenarios fardos waris son hallados en Huaca Pucllana: El Comercio. Lima-Perú.

Huertas, L. (1981). Culto a los muertos. En: La Religión en una Sociedad Rural Andina Siglo XVII. Pp.60. Lima.

Isbell, W. H. (2003). Sin Mallkis que adorar: los muertos Huari. En: Arqueológicas. Revista del Museo Nacional de arqueología, antropología e Historia del Perú. Vol. 26 Lima.

Kaulicke, P. (1997). La Muerte en el Antiguo Perú. Contextos y Conceptos Funerarios: Una Introducción. En: Boletín de Arqueología PUCP, Vol. 1, 1997, pp.7-54. Lima.

Kaulicke, P. (1997). Contextos Funerarios de Ancón. Esbozo de una síntesis analítica. PUCP Primera edición. Lima.

Kaulicke, P. (2000). Memoria y Muerte en el Perú Antiguo. PUCP Primera edición.

Kaulicke, P. (2000). La sombra de Pachacamac: Huari en la Costa Central. Boletín de Arqueología PUCP № 4, pp. 313-358. Lima, Perú.

Kaulicke, P. (2001). Vivir con los ancestros en el Antiguo Perú. En: La Memoria de los Ancestros. Luis Millones y Wilfredo Kapsoli (Compil.). Universidad Ricardo Palma Editorial Universitaria, pp. 25-61.

Maquera E. Esteban M. Álvarez M., González L.M., Pedemonte V., Silva E, Paz G. (2015). Arquitectura, Vida y Muerte en Lima entre los años 600 a 1000 d.C. Nuevos alcances desde la zona arqueológica Catalina Huanca. En: Arkinka N²34: 92-101.

Ortiz, A. (2011). "Los procesos tafonómicos en la formación de los depósitos funerarios" Estrat critic 5 Vol. 1, pp: 452-460. Universidad Autónoma de Barcelona - España. 
Paz, G. (2016). "Instrumentos para la vida, ofrendas para la muerte: Un estudio del ajuar funerario Wari de Huaca Pucllana" En: Revista de investigación Arqueología y Sociedad $\mathrm{N}^{\circ} 31$ de la UNMSM.

Pozzi-Escot, D.; Ángeles, R. (2005). Un Fardo Funerario en Huaca Malena, valle de Asia. En: Corriente Arqueológica, Muerte y Evidencias Funerarias en los Andes Centrales: Avances y Perspectivas, pp. 119-148. Lima.

Ravines, R. (1977). Prácticas Funerarias en Ancón (primera parte). En: Revista del Museo Nacional Lima Nº 43 INC T.XLIII 1977, pp. 327-397.

Ravines, R. (1981). Prácticas Funerarias en Ancón (segunda parte). En: Revista del Museo Nacional Lima № 45 INC T.XLV 1981, pp: 89-166. Lima

Segura, R. (1997). Contextos Funerarios A Fines del Horizonte Medio en la Necrópolis de Ancón, Perspectivas de Análisis e Investigación. Boletín de Arqueología PUCP, Vol. 1, pp. 241-251. Pontificia Universidad Católica del Perú, Lima.

Trivelli, C. (2008). Hallan una tumba intacta Wari en Huaca Pucllana. El Comercio. Perú.

Valladolid Clide. (2000). "Proceso de Desenfardelamiento del Fardo Funerario $\mathrm{N}^{\circ}$ 22 de Huallamarca" En: Arqueológicas Nㅜ24. Museo Nacional de Arqueología Antrpología e Historia del Perú. Publicación del Instituto de Investigaciones Antropológicas. Instituto Nacional de Cultura Lima. 